\title{
Self-dissociation of polar molecules in a confined infrared vacuum
}

\author{
Johan F. Triana ${ }^{1}$ and Felipe Herrera ${ }^{1,2}$ \\ ${ }^{1}$ Department of Physics, Universidad de Santiago de Chile, Avenida Ecuador 3493, Santiago, Chile. ${ }^{*}$ \\ ${ }^{2}$ Millennium Institute for Research in Optics (MIRO), Concepción, Chile ${ }^{\dagger}$
}

(Dated: July 23, 2020)

\begin{abstract}
Strong and ultrastrong light-matter interaction of molecular systems in infrared (IR) cavities is a promising tool for manipulating and controlling chemical reactivity and light emission. We study the infrared photodissociation dynamics of a single hydrogen fluoride (HF) molecule in a single-mode cavity vacuum, and compare it with the case of strong $\mathrm{cw}$ laser driving. Using the multiconfigurational time-dependent Hartree (MCTDH) method, we show that in the absence of additional IR sources, a single cavity mode can efficiently dissociate a polar diatomic molecule prepared in the ground vibrational level. For the HF molecule, we predict dissociation probabilities of up to $20 \%$ in less than $200 \mathrm{fs}$, for a vacuum field that is resonant with the fundamental vibration frequency at the onset of the ultrastrong coupling regime. The mean cavity photon number remains strongly constrained to a few dozen photons throughout the dissociation process. In contrast, similar dissociation rates can only be achieved in free space using resonant laser fields with intensities not smaller than $10^{14} \mathrm{~W} / \mathrm{cm}^{2}$. Our work highlights the fundamental differences that can be expected for reactive dynamical processes inside infrared cavities, in comparison with commonly studied cavity-free scenarios.
\end{abstract}

Molecular photodissociation with intense laser fields has been largely studied without considering the quantum nature of the electromagnetic field $[1,2]$. The research focus has been placed on the anharmonicity of the potential energy surfaces [3], the spectral properties of the driving laser fields [4], and the influence of the vibrational reservoir [5]. Several works have demonstrated how to break molecular bonds with infrared radiation via multiphoton excitation $[4,6,7]$, using chirped pulses [8-11] or combining a specific number of laser pulses [12-14]. Infrared dissociation requires high laser intensities, especially when the molecular system is initially in its ground vibrational state [4]. One alternative to avoid high laser intensities is to implement negative chirped pulses, where the carrier frequency is time-dependent and decreases with time, such that the pulse adapts to the vibrational anharmonicity. Dissociation probabilities with chirped pulses can be higher than with monochromatic lasers, for the same field intensity [8]. Laser pulses can be further optimized to dissociate molecular bonds with minimal field intensities. Optimal laser fields combine a finite number of monochromatic and chirped pulses that adapt better to the vibrational anhalyrmonicity, giving the highest possible dissociation probabilities [13].

If the quantum nature of radiation field is considered inside a cavity, molecular vibrations can form vibrational polaritons under strong or ultrastrong coupling with the quantized cavity field. Recent experiments have demonstrated strong and ultrastrong coupling with molecular solids and liquids [15-24]. This has accelerated and intensified theoretical efforts to understand the molecular and photonic properties of hybrid light-matter vibrational polariton states [25-29]. It was recently shown in Ref. [29], that the vibrations can become self-excited by interacting with a confined cavity field at the onset of ultrastrong coupling, depending on the shape of the electric dipole function $\hat{d}(q)$ along the vibrational coordinate $q$. The effect is most pronounced for molecular species whose electric dipole moment is greatest above the equilibrium bond length $q_{\mathrm{e}}$. Hydrogen fluoride (HF) is a representative example of this class of polar molecules, which we denote polar-right.

In this work, we compare the photodissociation dynamics of a single HF molecule in its ground electronic state, subject to driving either by a strong cw infrared laser or by a single-mode infrared cavity vacuum. This extends previous studies focused on intracavity photodissociation via electronic excited states [30-32], which have shown that classical and quantum photodynamics involving excited electronic states are qualitatively similar. We also propose an experiment in which both molecular fragments and photons generated in the polaritonic system can be detected at the same time.

In what follows, we briefly formulate the model used, and describe the numerical method used to solve the time-dependent Schrödinger equation. Then, we analyze and compare classical and quantum calculations both in the strong and ultrastrong coupling regime and finally discuss an experimental implementation that can be used to test our predictions.

The bare molecular Hamiltonian in the BornOppenheimer approximation is modeled as $\hat{H}_{\mathrm{M}}=\hat{T}(q)+$ $\hat{V}(q)$, where $\hat{T}(q)$ is the nuclear molecular kinetic energy operator and $\hat{V}(q)$ is the molecular potential energy curve. In order to study light-matter interaction, we assume in the semi-classical framework a cw electric field given by $\overrightarrow{\mathcal{E}}(t)=\mathcal{E}_{\mathrm{C}} \sin \left(\omega_{\mathrm{c}} t\right) \hat{\mathbf{e}}$, where $\mathcal{E}_{\mathrm{C}}$ is the electric field amplitude oriented along $\hat{\mathbf{e}}$ and $\omega_{\mathrm{c}}$ is the carrier 


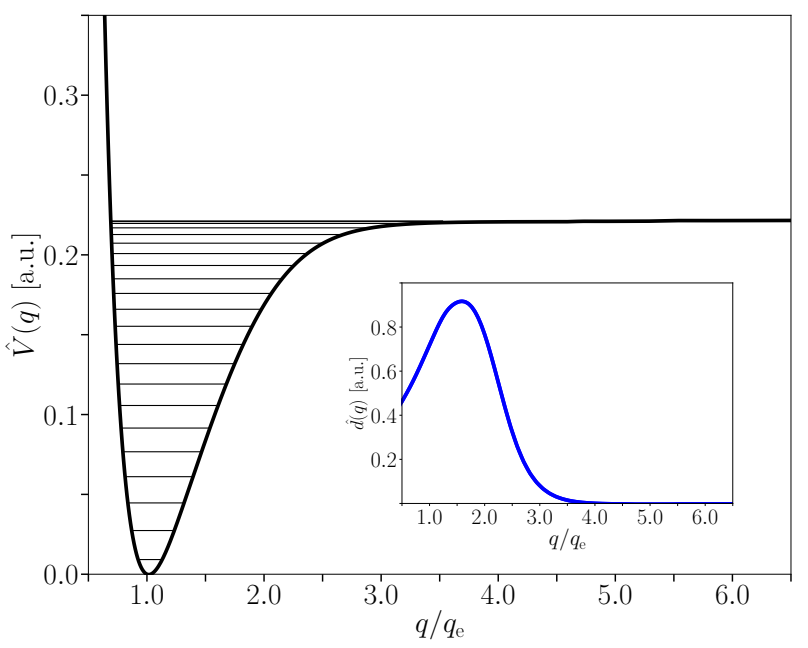

FIG. 1. ab initio potential energy curve $\hat{V}(q)$ of the electronic ground state $\mathrm{X}^{1} \Sigma^{+}$for $\mathrm{HF}$ molecules. The bond length $q$ is in units of the equilibrium distance $q_{\mathrm{e}}$. The energies of the 22 vibrational bound states are also shown. The inset shows the electric dipole function $\hat{d}(q)$ along the scaled bond distance.

frequency. In this form, the semi-classical light-matter Hamiltonian is given by

$$
\hat{H}_{\mathrm{sc}}=\hat{H}_{\mathrm{M}}(q)-\vec{d}(q) \cdot \overrightarrow{\mathcal{E}}(t),
$$

where $\vec{d}(q)$ is the molecular dipole moment function and $\overrightarrow{\mathcal{E}}(t)$ is the electric field.

In the quantum case, the electric field $\overrightarrow{\mathcal{E}}(t)$ is quantized $[31,33]$. We assume the electric field mode confined in an optical cavity with frequency $\omega_{\mathrm{c}}$. In this form, the electromagnetic mode is represented by a quantum harmonic oscillator with unit mass. As a basis set, we use Fock states $|n\rangle$ in the coordinate representation [34]. The light-matter Hamiltonian is thus given by

$$
\hat{H}=\hat{H}_{\mathrm{M}}(q)-\frac{1}{2} \frac{\partial^{2}}{\partial \hat{x}^{2}}+\frac{1}{2} \omega_{\mathrm{c}}^{2} \hat{x}^{2}+\sqrt{2 \omega_{\mathrm{c}}} \mathcal{E}_{0} \hat{d}(q) \hat{x},
$$

where $\hat{x}$ corresponds the quadrature of the cavity mode and $\mathcal{E}_{0} \equiv \lambda_{g} \omega_{\mathrm{c}} / d_{10}=\mathcal{E}_{\mathrm{C}} / 2$, with $d_{10}=$ $\langle\nu=1|\hat{d}(q)| \nu=0\rangle$ and where $|\nu\rangle$ are vibrational states of the potential $\hat{V}(q)$. The dimensionless parameter $\lambda_{g}$ corresponds to the parametrized light-matter coupling strength of the coupling ratio $g / \omega_{\mathrm{c}}$ used in the ultrastrong coupling literature [35], with coupling strength $g=$ $d_{10} \mathcal{E}_{0}$. The Hamiltonians in Eqs. (1) and (2) include counter rotating terms, independently of the coupling stregth $\lambda_{g}$. In a consistent multipolar formulation of non-covariant quantum electrodynamics [36], selfdipole terms do not depend on the cavity vacuum amplitude, and can be included as part of the vibrational Hamiltonian.

We perform numerical dynamics simulations using the multi-configurational time-dependent Hartree (MCTDH) method [37, 38]. MCTDH allows to implement the system Hamiltonians in Eqs. (1) and (2) directly to solve the nuclear time-dependent Schrödinger equation, i.e., semiclassical and quantum photodynamics are implemented using the same numerical propagation method. Further, MCTDH allows to perform projections of the molecular vibrational states onto the timedependent wavefunction and calculate the expectation values of any molecular or photonic operator $\hat{O}$ in the form $\langle\Psi(t)|\hat{O}| \Psi(t)\rangle$. The molecular degree of freedom $q$ corresponding to the bond distance between the hydrogen and fluoride atoms is defined in a sin-DVR primitive basis with $N_{q}=601$ grid points for bond distances between $q=0.5$ a.u. and $q=12.5$ a.u.. In the quantum case, i.e., when HF molecule is coupled to a quantized electric field [see Eq. (2)], the coordinate $x$ represents the cavity mode quadrature and it is described by a HO-DVR primitive basis functions with $N_{x}=481$ grid points between $x=-90.0$ and $x=90.0$, which is large enough to allocate all Fock states involved in the dynamics. Additional technical details of the MCTDH method and package can be found in Refs. [37] and [38].

For concreteness, in Fig. 1 we show the potential energy curve $\hat{V}(q)$ and electric dipole moment function $\hat{d}(q)$ of the HF molecule for the electronic ground state $\left(\mathrm{X}^{1} \Sigma^{+}\right)$, computed using a complete active space (CAS) of the lowest electronic state by a multi-configurational self-consistent field (MCSCF) method, followed by the multireference configuration interaction (MRCI) method, using Dunning's correlation consistent basis set with diffuse functions aug-cc-pVQZ, in the electronic structure package MOLPRO.

The initial wavefunction of the HF molecule corresponds to the vibrational ground state $\left|\Psi_{0}\right\rangle=|\nu=0\rangle$ in both semiclassical and quantum dynamics calculations. For the quantum case, the initial wavefunction is the direct product between the vibrational ground state and the cavity in the vacuum state $\left|\Psi_{0}\right\rangle=|\nu=0\rangle \otimes|0\rangle$, i.e., vibrationless molecule and blackbody radiation at $300 \mathrm{~K}$. The laser and cavity frequencies $\omega_{\mathrm{c}}$ are set equal to the fundamental vibration frequency $\omega_{10}=[E(\nu=$ 1) $-E(\nu=0)] / \hbar$.

In Fig. 2a, we show that using a classical laser field or a quantized vacuum for dissociation can result in different bond length dynamics. Although the mean bond distance oscillates with the same rate in the classical case (dashed black line) and the quantum case (blue line), the oscillation amplitudes are different, i.e., the vibrational excitation levels are not equivalent.

In the classical case, the mean bond distance of HF can reach up to $\langle\hat{q}\rangle=1.15 q_{\mathrm{e}}$ for a coupling strength $\lambda_{g}=0.02$, which in free space corresponds to the laser intensity $I_{0}=2.8 \times 10^{12} \mathrm{~W} / \mathrm{cm}^{2}$. The value $1.15 q_{\mathrm{e}}$ is close to average bond length of the third and fourth excited vibrational states, which indicates that the total wavefunction has contributions mostly of 

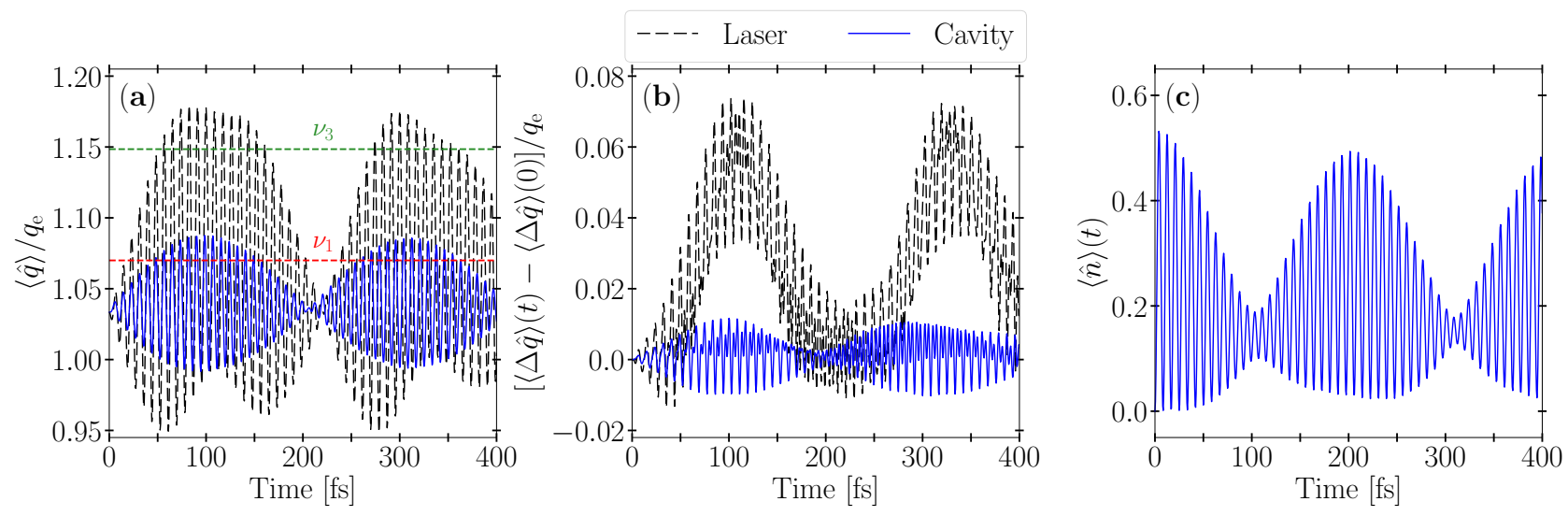

FIG. 2. (a) Mean bond length of an HF molecule coupled to a classical electric field (dashed black) and to a quantized electric field (blue), as a function of time. Horizontal dashed lines represent the bond lengths of the excited vibrational levels $\nu=1$ (red) and $\nu=3$ (green). (b) Spread of the bond distance $\langle\Delta \hat{q}\rangle(t)$ as a function of time. (c) Evolution of the mean intracavity photon number $\langle\hat{n}\rangle$. In all panels the coupling strength is set to $\lambda_{g}=0.02$, which in free space corresponds to the laser intensity $I_{0}=2.8 \times 10^{12} \mathrm{~W} / \mathrm{cm}^{2}$, for $\omega_{\mathrm{c}}=\omega_{10}$.

the first five vibrational levels of the ground electronic state during the propagation time. Excited vibrational states higher than $|\nu=5\rangle$ are not populated because the laser frequency is not resonant to higher vibrational transitions, and the intensity is not strong enough to induce significant ac-Stark shifts.

In the quantum case, the bond length $\langle\hat{q}\rangle$ of HF (blue line in Fig. 2a) oscillates around the bond distance of the ground vibrational state $\left(1.03 q_{\mathrm{e}}\right)$, with much smaller deviations $\langle\Delta \hat{q}\rangle \equiv \sqrt{\left\langle\hat{q}^{2}\right\rangle-\langle\hat{q}\rangle^{2}}<0.02 q_{\mathrm{e}}$, than the classical case (see Fig. 2b). In the quantum case the total wavefunction has contributions mostly of the lowest three vibrational states throughout the wavepacket evolution. The results show a resonant exchange of energy between the $0-1$ vibrational excitation and the cavity vacuum. Detailed inspection shows that the second excited vibrational state does not contribute to the polariton wavepacket. This can also be seen in the evolution of the mean cavity photon number $\langle\hat{n}\rangle$ in Fig. 2c, which oscillates between 0 and 0.5 , as expected for a quantized vacuum coupled to a two-level material system.

This scenario should be compared with the classical case, in which the $\mathrm{cw}$ laser field represents a constant energy supply for the HF molecule. Under intense laser excitation the vibrational population of the second excited vibrational level can reach up to $41 \%$ of the vibrational wavepacket. For the coupling strengths and field frequencies considered in Figs. 1 and 2, the population remains confined to the bound vibrational manifold, both in the classical and quantum cases. No photodissociation occurs.

Whereas IR photodynamics in vibrational strong coupling follows the physical intuition, the intracavity evolution is not straightforward for stronger light-matter interaction. It has been recently shown [29] that under ultrastrong coupling, both transition and permanent dipole moments are needed to properly describe lightmatter dynamics. The model in Ref. [29] was successfully used to interpret short-time excited polariton absorption peaks of sodium nitroprusside in Fabry-Perot cavities [24]. One unexpected feature of polar-right molecules (e.g., HF) in infrared cavities, is that highly excited vibrational states can become populated over sub-picosecond timescales, even when the dynamics is initialized in the cavity-free diabatic product between the ground vibrational state and the cavity vacuum $\left|\Psi_{0}\right\rangle=$ $|\nu=0\rangle \otimes|0\rangle$.

In Figure 3a we show the mean bond length for classical (dashed black line) and quantum (blue line) photodynamics, for a stronger coupling $\lambda_{g}=0.20$ or laser intensity $I_{0}=2.8 \times 10^{14} \mathrm{~W} / \mathrm{cm}^{2}$. In the classical case, the bond length $\langle\hat{q}\rangle$ reaches values close to the vibrational state $|\nu=20\rangle$, which is the second bound state below the dissociation threshold. For a dissociation probability given by

$$
P_{\text {diss }}=1-\sum_{\nu=0}^{21}|\langle\nu \mid \Psi(t)\rangle|^{2},
$$

the probability to break the HF bond with a resonant laser field is about $40 \%$, for the intensity considered, as shown in Fig. 3b. Note that more efficient laser dissociation strategies are known [3, 4, 9, 13]. Our goal is to compare photodissociation with $\mathrm{cw}$ laser and quantized vacuum fields.

In the case of quantum photodynamics, the higher values of $\langle\hat{q}\rangle$ relative to the classical case at early times occur because the initial state is not an eigenstate of the total Hamiltonian. Therefore, both molecular 

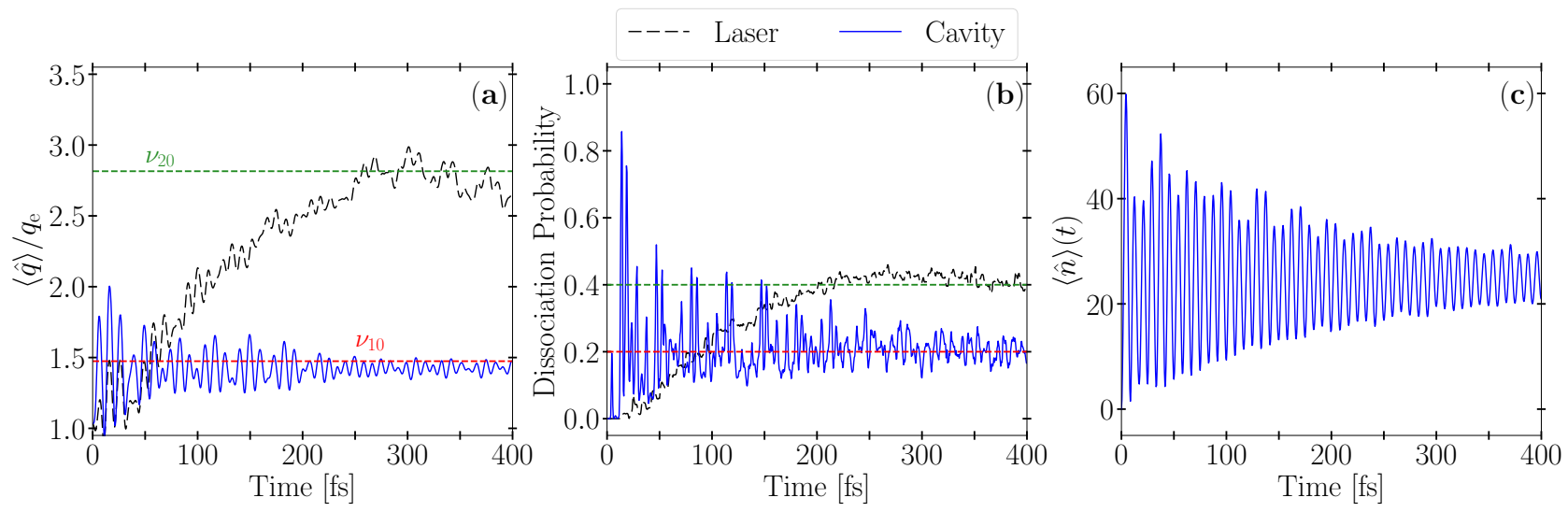

FIG. 3. (a) Mean bond length of an HF molecule coupled to a classical electric field (dashed black) and to a quantized electric field (blue), as a function of time. Horizontal dashed lines are the bond lengths of the excited vibrational levels $\nu=10$ (dashed red) and $\nu=20$ (dashed green). (b) Dissociation probability $P_{\text {diss }}$ for HF as a function of time. Horizontal dashed lines correspond to probabilities 0.2 (dashed red) and 0.4 (dashed green). (c) Evolution of the mean intracavity photon number $\langle\hat{n}\rangle$. In all panels the coupling strength is set to $\lambda_{g}=0.20$, corresponding to the free-space intensity $I_{0}=2.8 \times 10^{14} \mathrm{~W} / \mathrm{cm}^{2}$, for $\omega_{\mathrm{c}}=\omega_{10}$.

and radiation field excitations (photons) are quickly generated. Cavity-molecule energy exchange occurs as in the strong coupling regime, but without a welldefined rate. Fig. 3a shows that the bond length of HF is close to that of the 10-th excited vibrational state. The vibrational contribution to the total polariton wavepacket is shared between bound and unbound vibrational levels. The finite unbound population gives the dissociation probability in Fig. 3b. In other words, a polar-right molecular vibration coupled to a quantized field in the ultrastrong coupling has a finite probability to self-dissociate. The HF bond can break without infrared $\mathrm{cw}$ or pulse laser driving, and also without any initial photon inside the cavity. For the HF molecule, the dissociation probability is about $20 \%$ for $t>200 \mathrm{fs}$. Figure $3 \mathrm{c}$ shows that the number of intracavity photons generated in the light-matter interaction remains bound to a time-average of $\langle\hat{n}\rangle \approx 25$, throughout the evolution. These photons can be in principle detected as they leak out of the cavity volume into the far field [29].

It should be possible to simultaneously measure bond breaking and spontaneous IR photo-emission. By combining ideas from previously proposed detection schemes [29,39], we envision an experimental setup that initializes light-matter interaction and enables molecular and photonic detection. The setup would involve the generation of a diabatic product state with a define number of vibrational excitations and a define cavity photon number. Considering that the system evolves initially into a vibrational polariton wave packet, velocity mapping detectors for molecular fragmentation can be placed orthogonal to the cavity axis, to detect atomic fragments. Single photon detectors localized behind the cavity mirrors can detect the emitted photons.

In summary, we describe the differences between photodissociation of a single HF molecule subject to driving either by a cw infrared laser or by a single-mode infrared cavity vacuum in the strong and ultrastrong coupling regimes. We find dramatic differences in the resulting photodynamics, in contrast to previous suggestions that classical and quantum photodynamics are equivalent $[30,32]$. By comparing the mean bond distance $\langle\hat{q}\rangle$ and projections of the bound vibrational states $|\nu\rangle$ of the molecular potential over the total vibrational polariton wavefunction $|\Psi(t)\rangle$, we show that by coupling polar-right molecular species with vacuum fields, it is possible to obtain both emitted photons and molecular fragments without any other external force or pump-probe schemes. We also describe a possible experimental scheme to detect the dissociated atoms of the HF molecule and photons emitted at the same time. The results described here can be generalized to other alkali halide species. We focus here on the single-molecule case, but it is known that polaritonic dynamical features are transferable to the many-body regime [40].

\section{ACKNOWLEDGMENTS}

J.F.T. is supported by ANID through the Postdoctoral Fellowship Grant No. 3200565. F.H. is supported by ANID through grants REDES ETAPA INICIAL, Convocatoria 2017 No. REDI 170423, and FONDECYT Regular No. 1181743. Both authors thank generous support of Iniciativa Científica Milenio (ICM) through the Millennium Institute for Research in Optics (MIRO). 
* johan.triana@usach.cl

† felipe.herrera.u@usach.cl

[1] F. Großmann. Theoretical Femtosecond Physics: Atoms and Molecules in Strong Laser Fields. Graduate Texts in Physics. Springer International Publishing, 2013.

[2] Alicia Palacios, José Luis Sanz-Vicario, and Fernando Martín. Theoretical methods for attosecond electron and nuclear dynamics: applications to the $\mathrm{h}_{2}$ molecule. Journal of Physics B: Atomic, Molecular and Optical Physics, 48(24):242001, 2015.

[3] M. E. Goggin and P. W. Milonni. Driven morse oscillator: Classical chaos, quantum theory, and photodissociation. Phys. Rev. A, 37:796-806, 1988.

[4] E.F. de Lima, E.C. Rosado, L.K. Castelano, and R. Egydio de Carvalho. Quantum-classical correspondence and the role of the dipole function in molecular dissociation. Physics Letters A, 378(36):2657 - 2663, 2014.

[5] Chandan Kumar Mondal, Pinaki Chaudhury, and S.P. Bhattacharyya. Photo-dissociation dynamics of a diatomic molecule: modelling of thermal and environmental effects. Chemical Physics Letters, 311(5):400 - 406, 1999.

[6] R. Duperrex and H. van den Bergh. Time resolved measurement of $\mathrm{CF}_{2}$ formation in the infrared multiphoton dissociation of $\mathrm{CF}_{2} \mathrm{HCl}$. The Journal of Chemical Physics, 71(9):3613-3619, 1979.

[7] D. S. Bomse, R. L. Woodin, and J. L. Beauchamp. Molecular activation with low-intensity $\mathrm{cw}$ infrared laser radiation. multiphoton dissociation of ions derived from diethyl ether. Journal of the American Chemical Society, 101(19):5503-5512, 1979.

[8] Szczepan Chelkowski, André D. Bandrauk, and Paul B. Corkum. Efficient molecular dissociation by a chirped ultrashort infrared laser pulse. Phys. Rev. Lett., 65:23552358, 1990.

[9] J T Lin, T L Lai, D S Chuu, and T F Jiang. Quantum dynamics of a diatomic molecule under chirped laser pulses. Journal of Physics B: Atomic, Molecular and Optical Physics, 31(4):L117-L126, 1998.

[10] Jian-Min Yuan and Wing-Ki Liu. Classical and quantum dynamics of chirped pulse dissociation of diatomic molecules. Phys. Rev. A, 57:1992-2001, 1998.

[11] Marcus, G., Zigler, A., and Friedland, L. Molecular vibrational ladder climbing using a sub-nanosecond chirped laser pulse. Europhys. Lett., 74(1):43-48, 2006.

[12] D.W. Noid and J.R. Stine. Infrared multiphoton dissociation with one and two lasers. Chemical Physics Letters, 65(1):153 - 157, 1979.

[13] Matjaž Kaluža, James T. Muckerman, Peter Gross, and Herschel Rabitz. Optimally controlled five-laser infrared multiphoton dissociation of HF. The Journal of Chemical Physics, 100(6):4211-4228, 1994.

[14] M.V. Korolkov, J. Manz, and G.K. Paramonov. Theory of ultrafast laser control for state-selective dynamics of diatomic molecules in the ground electronic state: vibrational excitation, dissociation, spatial squeezing and association. Chemical Physics, 217(2):341 - 374, 1997. Dynamics of Driven Quantum Systems.

[15] J. P. Long and B. S. Simpkins. Coherent coupling between a molecular vibration and fabry-perot optical cavity to give hybridized states in the strong coupling limit. ACS Photonics, 2(1):130-136, 2015.

[16] Omree Kapon, Rena Yitzhari, Alexander Palatnik, and Yaakov R. Tischler. Vibrational Strong Light-Matter Coupling Using a Wavelength-Tunable Mid-infrared Open Microcavity. Journal of Physical Chemistry C, 121(34):18845-18853, 2017.

[17] Merav Muallem, Alexander Palatnik, Gilbert D. Nessim, and Yaakov R. Tischler. Strong Light-Matter Coupling and Hybridization of Molecular Vibrations in a LowLoss Infrared Microcavity. Journal of Physical Chemistry Letters, 7(11):2002-2008, 2016.

[18] Thibault Chervy, Anoop Thomas, Elias Akiki, Robrecht M. A. Vergauwe, Atef Shalabney, Jino George, Eloïse Devaux, James A. Hutchison, Cyriaque Genet, and Thomas W. Ebbesen. Vibro-Polaritonic IR Emission in the Strong Coupling Regime. ACS Photonics, 5(1):217224, 2018.

[19] Jino George, Atef Shalabney, James A. Hutchison, Cyriaque Genet, and Thomas W. Ebbesen. Liquidphase vibrational strong coupling. Journal of Physical Chemistry Letters, 6(6):1027-1031, 2015.

[20] Jino George, Thibault Chervy, Atef Shalabney, Elö̈se Devaux, Hidefumi Hiura, Cyriaque Genet, and Thomas W. Ebbesen. Multiple Rabi Splittings under Ultrastrong Vibrational Coupling. Physical Review Letters, 117(15):153601, 2016.

[21] Wonmi Ahn, Igor Vurgaftman, Adam D. Dunkelberger, Jeffrey C. Owrutsky, and Blake S. Simpkins. Vibrational Strong Coupling Controlled by Spatial Distribution of Molecules within the Optical Cavity. ACS Photonics, 5(1):158-166, 2018.

[22] A. Thomas, L. Lethuillier-Karl, K. Nagarajan, R. M. A. Vergauwe, J. George, T. Chervy, A. Shalabney, E. Devaux, C. Genet, J. Moran, and T. W. Ebbesen. Tilting a ground-state reactivity landscape by vibrational strong coupling. Science, 363(6427):615-619, 2019.

[23] Felipe Herrera and Jeffrey Owrutsky. Molecular polaritons for controlling chemistry with quantum optics. The Journal of Chemical Physics, 152(10):100902, 2020.

[24] Andrea B. Grafton, Adam D. Dunkelberger, Blake S. Simpkins, Johan F. Triana, Federico J. Hernandez, Felipe Herrera, and Jeff Owrutsky. Excited-state vibrationpolariton transitions and dynamics in nitroprusside. Chemrxiv.12518555, 2020.

[25] Luis A. Martínez-Martínez, Raphael F. Ribeiro, Jorge Campos-González-Angulo, and Joel Yuen-Zhou. Can Ultrastrong Coupling Change Ground-State Chemical Reactions? ACS Photonics, 5(1):167-176, 2018.

[26] Javier del Pino, Johannes Feist, and F. J. GarciaVidal. Signatures of vibrational strong coupling in raman scattering. The Journal of Physical Chemistry C, 119(52):29132-29137, 122015.

[27] Artem Strashko and Jonathan Keeling. Raman scattering with strongly coupled vibron-polaritons. Phys. Rev. A, 94:023843, 2016.

[28] Federico Hernández and Felipe Herrera. Multilevel quantum rabi model for anharmonic vibrational polaritons. The Journal of Chemical Physics, 151:144116, 2019.

[29] Johan F. Triana, Federico J. Hernández, and Felipe Herrera. The shape of the electric dipole function determines the sub-picosecond dynamics of anharmonic vibrational polaritons. The Journal of Chemical Physics, 152(23):234111, 2020. 
[30] Markus Kowalewski, Kochise Bennett, and Shaul Mukamel. Cavity femtochemistry; manipulating nonadiabatic dynamics at avoided crossings. J. Phys. Chem. Lett., 7(11):2050-2054, 2016.

[31] Markus Kowalewski, Kochise Bennett, and Shaul Mukamel. Non-adiabatic dynamics of molecules in optical cavities. J. Chem. Phys., 144(5), 2016.

[32] András Csehi, Gábor J. Halász, Lorenz S. Cederbaum, and Ágnes Vibók. Intrinsic and light-induced nonadiabatic phenomena in the NaI molecule. Phys. Chem. Chem. Phys., 19:19656-19664, 2017.

[33] Johan F. Triana, Daniel Peláez, and José Luis SanzVicario. Entangled photonic-nuclear molecular dynamics of lif in quantum optical cavities. The Journal of Physical Chemistry A, 122(8):2266-2278, 2018.

[34] C. Gerry and P. Knight. Introductory quantum optics. Cambridge University Press, Cambridge, 2005.

[35] Wolfgang Demtröder. Molecular physics: theoretical principles and experimental methods. John Wiley \& Sons, 2008.

[36] David L. Andrews, Garth A. Jones, A. Salam, and R. Guy Woolley. Perspective: Quantum hamiltonians for optical interactions. The Journal of Chemical Physics, 148(4):040901, 2018.

[37] M.H. Beck, A. Jackle, G.A. Worth, and H.-D. Meyer. The multiconfiguration time-dependent hartree (mctdh) method: a highly efficient algorithm for propagating wavepackets. Physics Reports, 324(1):1 - 105, 2000.

[38] Graham Worth, Michael Beck, Andreas Jäckle, and Hans-Dieter Meyer. The MCTDH Package, Version 8.2, (2000). H.-D. Meyer, Version 8.3 (2002), Version 8.4 (2007). Current version: 8.4.18 (2019). See http://mctdh.uni-hd.de.

[39] Johan F. Triana and José Luis Sanz-Vicario. Revealing the presence of potential crossings in diatomics induced by quantum cavity radiation. Phys. Rev. Lett., 122:063603, Feb 2019.

[40] Felipe Herrera and Frank C. Spano. Absorption and photoluminescence in organic cavity QED. Physical Review A, 95(5):1-24, 2017. 